of growths about the face is urged on the assumption that noteworthy disfigurement is a necessary consequence of cutting operations. We know that such arguments are not valid when excision is made in the incipiency of the disease. In all other cases the question of cure is paramount.

A remedy for cancer that is lauded because its application does not disfigure the patient must possess curative properties superior to any other known treatment to entitle it to respectful consideration. In this case, the history of which has just been related, the $x$-ray proved to be not only a dismal failure as a cure, but it was positively harmful, both in wasting time and in stimulating the cancer's growth.

Bodine's method of using cocain locally enables the surgeon to avoid the objection to, and the dangers and disagreeable features of, general anesthesia, and promises the practical certainty of a painless operation. In - my patient's case a positive history of attacks of true angina pectoris was an important factor. It seemed advisable at the operation of April 6, 1904, to give the patient a hypodermic of morphin before injection of the cocain solution was begun, because it is the systemic antidote to cocain and also the most potent agent we may employ in angina pectoris. This precaution was probably unnecessary. In the second cutting operation the use of morphin was omitted. In both instances no untoward cardiac symptoms were observed either during or subsequent to the operation.

58 West Fortieth Street.

\section{A CASE OF SYPHILITIC DACTYLITIS OF THE TOE.}

ASTLEY PASTON COOPER ASHHURST, M.D.

Surgeon to the Out-Patient Department of the Episcopal Hospital. PHILADELPHIA.

Patient.-R. E.; aged 40, applied to the surgical dispensary of the Episcopal Hospital April 20, 1904, for treatment of his left foot.

Examination.-The foot was moderately swollen, with two areas of superficial ulceration on the dorsum, and with the middle toe the seat of a swelling so typical of syphilitic dactylitjs as seen in the fingers, that the specific nature of the lesions was readily determined. The patient stated that the ulcerations of the dorsum of the foot had persisted more or less in their present form for a period of eighteen months and that the toe had been in its present state for about six months. He had tried various salves and ointments, but so far no form of application had proved of any avail. On further inquiry it was learned that twelve years previously the man had had a sore on the penis, which, although accompanied by suppurative inguinal buboes, was nevertheless followed later on by an evanescent skin rash. Antisyphilitic treatment had been employed for about six weeks. The accompanying illustration, kindly made for me by Dr. R. S. Hooker, shows very well the appearance of the foot and toe when the patient came under my treatment.

Treatment and Result.-The ulcers were dressed locally with dilute citrin ointment, and iodid of potash was administered internally. Improvement was rapid, the ulcers healed, and the dactylitis disappeared, so that in the spring of 1904 the foot was practically normal. I heard from this patient in November, 1905 , and learned that he has taken the potassium more or less regularly ever since being under my care; he says he learned by experience that soon after stopping the medicine he was prone to develop ulcers or sores in some part of his body. His foot has given him no further trouble.

Syphilitic dactylitis was first accurately described by Dr. R. W. Taylor, ${ }^{1}$ of New York, in 1871. The affection

1. Amer. Jour. Syph. and Derm., 1871, vol, i1, p. 1. had been alluded to previously by one or two writers, but it had never before been fully studied. When Van Harlingen, in 1882, wrote the article on "Syphilis" for the International Encyclopædia of Surgery, he could find less than twenty-four cases of the affection recorded. It occurs in two varieties: The more common variety affects the subcutaneous tissues and the fibrous structures of the joints; the other arises in the periosteum and the bones. It does not, as a rule, make its appearance until five to fifteen years after the primary lesion.



Syphilltic Dactylitis.

It appears to be distinctly rarer in the toes than in the fingers. I have observed two cases of syphilitic dactylitis of the finger in the dispensary of the Episcopal Hospital, one being a manifestation of hereditary syphilis in a child, the other occurring in an adult. In the finger it is nearly always the proximal phalanx that is affected; but in the toe, as is evidenced in the case herewith recorded, the swelling usually extends throughout the whole length of the affected digit. As remarked before, the appearance is characteristic, and even if no syphilitic history could be elicited I think the true cause of the lesions would be readily determined.

\section{OSTEOMA OF THE OUTER SURFACE OF THE ULNA.}

C. B. CLAPP, M.D

Surgeon in charge, Wabash Employés' Hyospital. MOBERLY, MO.

Patient.-J. S. H., aged 28, section laborer, when about 10 years of age noticed a small lump on the outer surface, near the lower end of the right ulna, which has slowly yet constantly grown until the present time. He does not remember any traumatism to this part at any time.

Description of Tumor.-The tumor itself measures $71 / 2$ inches in circumference; its surface is rough and jagged, resembling the caulifiower somewhat in appearance. The radiograph (Fig. 1), which was taken by me before the operation, shows clearly the usual shortening of the shaft of the ulna and the drawing away of its articular surface from the wrist joint. It also shows the peculiar curving of the shafts of both the bones of the forearm, compensating, in part, for this development, and also the malposition of the distal extremity of the ulna. Since 
the operation the distal piece has acquired a more normal position and will doubtless adapt itself more closely to the wrist joint in time and thereby afford material strength.

Remarls.-It is interesting to note that this man has always done the heaviest kind of manual labor, using the shovel constantly for days, driving spikes with a heavy sledge, lifting

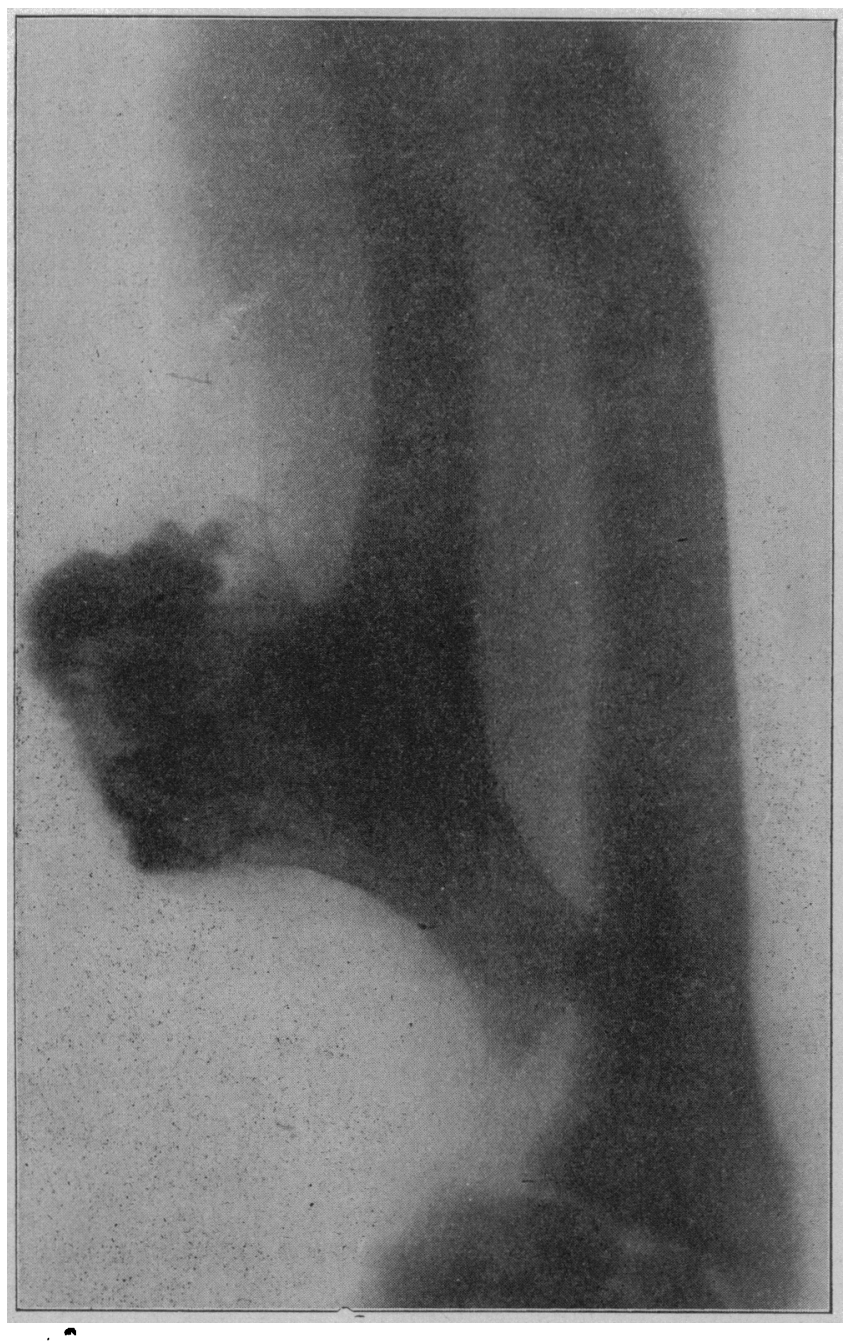

Fig. 1.-Showing the shortening of ulna and malposition of articular surface.

steel rails, handling ties, and all work usually done by a man on the section, and at no time, either before or since the operation, has he noticed that this wrist or arm tires before the other, or is weaker than the other.

\section{New Instrument}

\section{A NEW ANOSCOPE.}

\section{T. CHITTENDEN HILL, M.D.}

Surgeon to the Rectal Department of the Boston Dispensary. Boston.

This new instrument, which I devised for a special case, has proved of such general utility in the treatment of affections of the lower rectum and anal canal that a brief description of it may be of interest:

For examinations and treatment of the rectum proper and pelvic colon the round tubes of Kelley and the later pneumatic modifications of these leave nothing to be desired but for the treatment of ulcerations, abrasions, new growths, etc., of the anal canal the specula in common use have not at all times been satisfactory. A diagnosis can be made by digital examination, but it is often important to have a good view of the lesion in making local treatments. With this simple instrument I have treated many patients who otherwise would have been obliged to undergo general anesthesia.

It consists, as the cut shows, of a straight glass tube with an oval window in the side. The lower end is rounded and punctured by a small hole which allows air dilatation of the rectum. The handle is detachable and to prevent eracking of the glass it should be removed when the instrument is sterilized.

METIIOD OF USE.

With the patient either on the elbows and knees or in the right semi-prone position the distal end is smeared with vaselin and pressed firmly over the anal orifice. Then as the patient strains down the instrument slips easily past the sphineters.

Should there be an excess of vaselin about the window in the instrument it is wiped away with a cotton swab. By gently rotating and partially withdrawing the tube the whole anal circumference can now be thoroughly explored without discomfort to the patient.

ADVANTAGES,

The following advantages are claimed for this speculum over

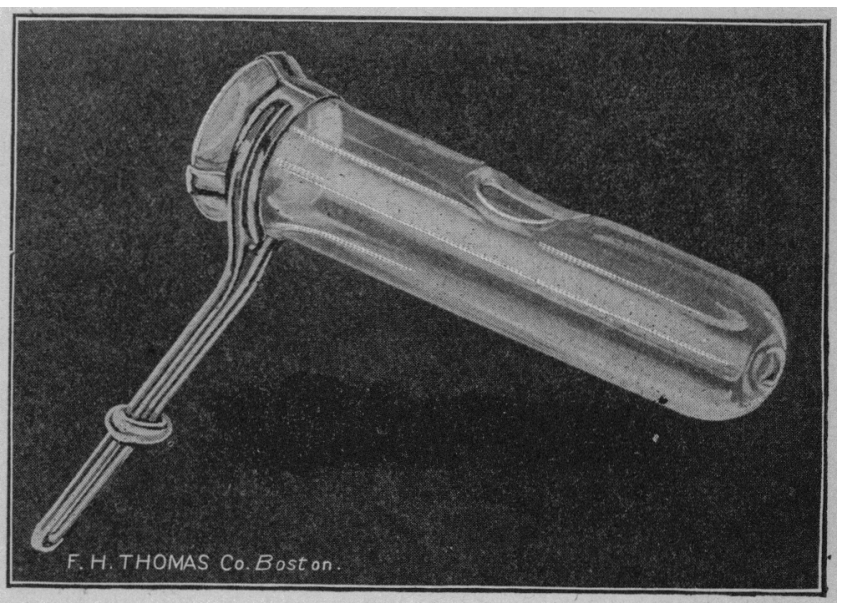

others now in use: The round tube is the most easily introduced when there is spasm of the sphincters, which is nearly always the case in disease in this locality. The edges of the oval window are so smooth that rotating and withdrawing it does not pinch the mucous membrane and thus cause painand what is more important, the mucous membrane anterior to the part being examined is firmly supported and thus a good view is obtained.

In the bivalve specula and the conical fenestrated ones with removable blades, the mucous membrane so prolapses into the instrument that a satisfactory examination is often impossible. Besides, their use is painful and they have to be reintroduced several times in order to examine thoroughly the whole of the anal canal.

When necessary for special cases the window may be enlarged, made smaller, or placed at a different level. The one in the cut, however, has proved suitable for nearly every case.

This instrument I now use almost exclusively when a speculum is required for the examination or treatment of the anal canal. And when it it recalled that more than half of the diseases of the rectum are in this locality its field of usefulness is readily appreciated.

Milk in Pulmonary Tuberculosis.-When any dyspepsia arises from the use of milk, Burton-Fanning advises adding 20 grains (an eggspoonful) of sodium bicarbonate to each tumblerful. The milk may also be given warm, or thickened with some farinaceous food, or it may have a little spirit added. It should not be swallowed in bulk, but should be slowly sipped. With these modifications milk usually agrees, even when drunk at meals with other food. A great deal of milk can be administered in the form of junket or other pudding, to wh.reh cream may be add zd.-Denver Medical Times. 